\title{
On the Optimality of the Greedy Solutions of the General Knapsack Problems
}

\author{
B. VIZVÁRI
}

Bilkent University, Dept. of Industrial Engineering, Ankara

\begin{abstract}
Summary: In this paper we submit a unified discussion of some closely related results which were achieved independently in number theory and integer programming, and we partially generalize them. In the unified discussion we treat together two problems where the greedy method has different characters, in the first one it is an internal-point algorithm, in the second one it is an outer-point method. We call a knapsack problem "pleasant" if the greedy solution is optimal for every right-hand side. A sufficient and two finite necessary and sufficient conditions for the pleasantness of a problem are discussed. The sufficient condition can be checked very easily. The paper is finished with an error analysis of some nonpleasant problems.
\end{abstract}

AMS 1980 Subject Classification:

Primary: $90 \mathrm{C} 10$

Key words: Combinatorial optimization, knapsack problems, greedy method.

\section{Introduction}

In combinatorial optimization the greedy method is defined for many problems. The following two questions are very frequently investigated in connection with the greedy method: when is the greedy solution optimal and if it fails to be optimal, how large its error can be in the objective function. These questions are answered generally in the case of problems where the greedy method is a so-called internal-point method, i.e., it goes through feasible solutions. However, MAGAZINE et al. give an answer in [4] for a special kind of the knapsack problem where the greedy method is an outer-point method, i.e., only the last point is feasible.

A special case of the same knapsack problem has been a tool in number theory in the investigations of the FroBenius and the post-stamp problems. Thus DJawadi has obtained independently in [1] some results similar to the main theorem of [4]. To obtain further results in the field of the post-stamp problem, MaRSTANDER has needed an analogous version of this theorem for a knapsack problem having an opposite objective function [5]. ZöLNER [7], JoHNSON and Kernigham [3] have given two different necessary and sufficient conditions for the optimality of the greedy solution at every right-hand-side in the special case considered by DJAWADI. A good summary of these number theoretic results is in [6, Ch. IV. and X.]. 
In this paper we give a unified discussion of all of these results and parth generalize them In Section 2 we define the knapsack problems and the greedy method and introduce some notations needed to the unified discussion. The necessary and sufficient conditions for the optimality of the greedy solution at every right-hand-side are given in Section 3. In the next section the reader finds a recursive sufficient condition for the same problem which can be checked very easily. An error analysis is given in the last section for the cases where the condition fails.

\section{The Knapsack Problems and the Greedy Method}

We deal with two different knapsack problems. Our aim is to give a unified discussion for them. Therefore we denote their coefficients in the same way. The two problems are

$$
\begin{aligned}
\max & \sum_{j=1}^{n} c_{j} x_{j} \\
& \sum_{j=1}^{n} a_{j} x_{j}=b \\
& x_{j} \in \mathbb{Z}_{+} \quad j=1, \ldots, n .
\end{aligned}
$$

and

$$
\begin{aligned}
\min & \sum_{j=1}^{n} c_{j} x_{j} \\
& \sum_{j=1}^{n} a_{j} x_{j}=b \\
& x_{j} \in \mathbb{Z}_{+} \quad j=1, \ldots, n .
\end{aligned}
$$

We assume the following for the coefficients in both problems

$$
c_{1}, \ldots, c_{n} \in \mathbb{Z}_{+}, a_{1}, \ldots, a_{n}, b \in \mathbb{N}
$$

and

$$
a_{1}=1
$$

Constraint (2.4) ensures that the greedy method defined below gives a feasible solution for every positive integer right-hand-side.

We emphasize that (2.1) and (2.2) are not two problems having the same coefficients but are two different problems and we denote their coefficients in 
She vime un Hherefore we mime in the case of Problem (2.1) that the variables are idexed, such that

$$
\frac{c_{j}}{a_{j}} \leq \frac{c_{j+1}}{a_{j+1}} \quad j=1, \ldots, n-1,
$$

and in the case of Problem (2.2) they are indexed, such that

$$
\frac{c_{j}}{a_{j}} \geq \frac{c_{j+1}}{a_{j+1}} \quad j=1, \ldots, n-1 .
$$

For the sake of the unified discussion, we introduce the ordering relations $\unrhd$ and $\unlhd$ defined by

$$
\unrhd=\left\{\begin{array}{ll}
\geq \\
\leq
\end{array} \text { and } \unlhd= \begin{cases}\leq & \text { in the case of Problem (2.1) } \\
\geq & \text { in the case of Problem (2.2). }\end{cases}\right.
$$

Assume that two values of the objective function belonging to different feasible solutions stand on the two sides of sign $\unlhd$ and the relation holds. Then the value standing on the right-hand-side of $\unlhd$, is at least as good as the other one. Therefore, one can read the formula " $u \unlhd v$ " as " $u$ is an impairment considering $v$ ", and the formula " $u \unrhd v$ " as " $u$ is an improvement considering $v$ ". With this notation the constraints (2.5) and (2.6) can be unified as

$$
\frac{c_{j}}{a_{j}} \unlhd \frac{c_{j+1}}{a_{j+1}} \quad j=1, \ldots, n-1
$$

Thus the reserve index order is a plausible evaluation of the variables. Hence the greedy solution of both problems is

$$
x_{j}^{g}=\left\lfloor\frac{b-\sum_{i=j+1}^{n} a_{i} x_{i}^{g}}{a_{j}}\right\rfloor j=n, n-1, \ldots, 1 .
$$

The vector $x^{g}$ is really a feasible solution of the problems as using constraint (2.4), we obtain from (2.8)

$$
x_{1}^{g}=\left\lfloor\frac{b-\sum_{i=2}^{n} a_{i} x_{i}^{g}}{a_{1}}\right\rfloor=b-\sum_{i=2}^{n} a_{i} x_{i}^{g} \in \mathbb{Z}_{+} .
$$

In the most important special case of Problem (2.1) $c_{1}=0$. Then it is equivalent with

$$
\begin{aligned}
\max & \sum_{j=2}^{n} c_{j} x_{j} \\
& \sum_{j=2}^{n} a_{j} x_{j} \leq b \\
& x_{j} \in \mathbb{Z}_{+}, \quad j=2, \ldots, n .
\end{aligned}
$$


Here the greeds method goes through on the points

$$
\sum_{i=j}^{n} e_{i} x_{i}^{g} j=n, n-1, \ldots, 2,
$$

where $e_{i}$ is the $i$-th unit vector, which is really an internal-point method improving from point to point the value of the objective function. In the same special case of Problem (2.2), it is not equivalent with a problem of type (2.9). Thus here the greedy method is an outer-point method.

As we investigate the problems for all possible right-hand-sides, we must consider the right-hand-side as a parameter of the problems. Similarly as we give a recursive sufficient condition for the optimality of the greedy solutions, the number of variables will also be a parameter. Thus the optimal value of the objective function and the value of the greedy solution are the functions of $n$ and $b$ and are denoted by $f(n, b)$ and $g(n, b)$, respectively. Thus

$$
g(n, b)=\sum_{j=1}^{n} c_{j} x_{j}^{g} .
$$

If at the same time we need more than one greedy solution, then we denote the solution defined in $(2.8)$ by $x_{j}^{g}(n, b)(j=1, \ldots, n)$ for the sake of unambiguity. Similarly we denote the optimal solutions by $x^{*}$ or $x_{j}^{*}(n, b)(j=1, \ldots, n)$, respectively.

We obtain from (2.8) that

$$
\text { if } 1 \leq j<k \leq n \text { and } a_{j} \geq a_{k} \text { then } x_{j}^{g}=0 .
$$

The main emphasis of this paper is the optimality of greedy solutions. Therefore it is reasonable to exclude any variables which are equal 0 in all greedy solutions, i.e. we claim that

$$
a_{1}<a_{2}<\ldots<a_{n} .
$$

The constraints concerning the coefficients, i.e., constraints (2.3), (2.4), (2.7) and $(2.10)$, are called regularity conditions.

Definition 2.1: A problem of the type (2.1) or (2.2) is pleasant if the greedy solution is optimal for every right-hand-side, i.e., $f(n, \cdot)$ and $g(n, \cdot)$ as the functions of only the right-hand-side are identical. A solution of a problem is pleasant if it is at the same time a greedy and an optimal solution.

\section{The Necessary and Sufficient Conditions of the Pleasantness of the Knapsack Problems}

In this section we give two finite necessary and sufficient conditions for the pleasantness of the knapsack problem. Both theorems give a finite set $B$ of right-hand-sides, such if we have for every $b \in B$ a pleasant solution, then the problem is pleasant. 
Theore'm 3.1: lisume that the regularity conditions are satisfied and $n \geq 2$.

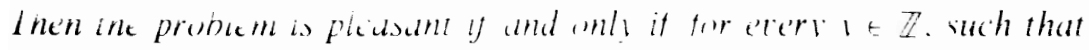

$$
a_{n+1}+1 \leq y \leq a_{n}+a_{n-1}-1
$$

we have

$$
f(n, y)=g(n, y) .
$$

Proof: The necessity is obvious. Suppose the existence of at least one righthand-side having no pleasant solution. Let

$$
m=\min \{y: f(n, y) \neq g(n, y)\} .
$$

Consider a solution $\boldsymbol{x}$ which is optimal or greedy, respectively. If $\boldsymbol{z} \in \mathbb{Z}_{+}^{n}$ and $\boldsymbol{z} \leq \boldsymbol{x}$, then $\boldsymbol{z}$ is an optimal or greedy solution respectively, for the appropriate right-hand-side. Let $\boldsymbol{x}^{*}$ be the optimal solution belonging to the right-handside $m$. Then it follows from the minimality property of $m$ that

$$
x_{j}^{g}(n, m)=0 \quad \text { or } \quad x_{j}^{*}=0, j=1, \ldots, n .
$$

Let

$$
k=\max \left\{j: x_{j}^{g}(n, m)>0\right\} .
$$

Hence we obtain that if $k<n$, then $m<a_{n}$ and

$$
x_{j}^{*}=0 \quad j=k, \ldots, n .
$$

The positivity of $m$ follows from its definition. Thus there is at least one index $i$, such that $i<k$ and

$$
x_{i}^{*}>0 \text {. }
$$

Consider the number $m-a_{i}$. According to the definition of $m$, the solution $x^{g}\left(n, m-a_{i}\right)$ is pleasant. We define the vector $z$ as follows

$$
z_{j}= \begin{cases}x_{j}^{g}\left(n, m-a_{i}\right) & \text { if } j \neq i \\ x_{i}^{g}\left(n, m-a_{i}\right)+1 & \text { if } j=i .\end{cases}
$$

Hence

$$
m=\left(m-a_{i}\right)+a_{i}=\sum_{j=1}^{n} a_{j} x_{j}^{g}\left(n, m-a_{i}\right)+a_{i}=\sum_{j=1}^{n} a_{j} z_{j} .
$$

From the choice of index $i$, it follows that vector $z$ is an optimal solution. Hence we obtain from (3.2) that

$$
z_{k}=x_{k}^{g}\left(n, m-a_{i}\right)=0 .
$$


The wetor $x^{q}(11, m$ a, ) a greeds solutwon, therefore

$$
m-u_{i}<i_{k} .
$$

Thus

$$
m \leq a_{k}+a_{i}-1 \leq a_{n}+a_{n-1}-1 .
$$

If $m<a_{n-1}$ then

$$
a_{n-1}<a_{n}+m \leq a_{n}+a_{n-1}-1
$$

and

$$
f\left(n, a_{n}+m\right) \neq g\left(n, a_{n}+m\right)
$$

as

$x_{n}^{g}(n, m)=0 ; x_{n}^{g}\left(n, a_{n}+m\right)=1 ; x_{j}^{g}(n, m)=x_{j}^{g}\left(n, m+a_{n}\right), j=1, \ldots, n-1$.

Thus the part formed by the first $n-1$ components of $\boldsymbol{x}^{g}\left(n, a_{n}+m\right)$ is not optimal.

Theorem 3.2: Assume that the regularity conditions are satisfied. Let

$$
m=\min \left\{y \in \mathbb{Z}_{+}: f(n, y) \neq g(n, y)\right\} .
$$

We define the indices $k$ and $p$ in the following way

$$
k=\max \left\{j: x_{j}^{g}(n, m)>0\right\}
$$

and

$$
p=\max \left\{j: \exists \boldsymbol{x}^{*}(n, m), x_{j}^{*}(n, m)>0\right\} .
$$

Then there are two indices: $q$ and $r$, such that

$$
p \geq q>r \geq 1, x_{r}^{g}\left(p, a_{k}\right)>0,
$$

and

$$
m=a_{q}+\sum_{j=q}^{p} a_{j} x_{j}^{g}\left(p, a_{k}\right) .
$$

Proof: From the choice of $m$ it is clear that $p<k$. First we prove that

$$
m<a_{p}+a_{p+1} \text {. }
$$

In the opposite case the greedy solution to $m-a_{p}$ is optimal and $x_{p+1}^{g}\left(n, m-a_{p}\right)>0$. Thus we have an optimal solution to $m$ where $x_{p+1}^{*}(n, m)>0$ which is a contradiction with the choice of $p$. Hence we obtain:

$$
g\left(n, m-a_{p}\right)=g\left(p, m-a_{p}\right)
$$

and thus

$$
f\left(n, m-a_{p}\right)=g\left(p, m-a_{p}\right) .
$$


If

$$
x_{j}^{g}(p, m) \geq x_{j}^{g}\left(p, a_{k}\right) \quad j=1, \ldots, p
$$

then clearly

$$
g(p, m)=g\left(p, m-a_{k}\right)+g\left(p, a_{k}\right) .
$$

If (3.5) does not hold, then let

$$
\mathrm{q}=\max \left\{j: 1 \leq j \leq p, x_{j}^{g}(p, m) \neq x_{j}^{g}\left(p, a_{k}\right)\right\} .
$$

It follows from the regularity conditions and from the definition of $k$ that

$$
m>a_{k} \text {. }
$$

Hence

$$
x_{q}^{g}(p, m)>x_{q}^{g}\left(p, a_{k}\right)
$$

As (3.5) does not hold, we have at least one index $r$, such that $r<q$ and

$$
0 \leq x_{r}^{g}(p, m)<x_{r}^{g}\left(p, a_{k}\right) .
$$

We define $w_{k, p, q}$ as

$$
w_{k, p, q}=a_{q}+\sum_{j=q}^{p} a_{j} x_{j}^{g}\left(p, a_{k}\right) .
$$

Hence

$$
a_{k}<w_{k, p, q} \leq m
$$

and furthermore the representation of $w_{k, p, q}$ in (3.6) belongs to the solution $\boldsymbol{x}^{g}\left(p, w_{k, p, q}\right)$, and

$$
x_{j}^{g}\left(p, w_{k, p, q}\right) \leq x_{j}^{g}(p, m) \quad j=1, \ldots, p .
$$

Hence

$$
g(p, m)=g\left(p, m-w_{k, p, q}\right)+g\left(p, w_{k, p, q}\right) .
$$

Our aim is to show that neither (3.5) nor $m>w_{k, p, q}$ can hold. Otherwise there is an integer $w$, such that

$$
a_{k} \leq w<m
$$

and

$$
g(p, m)=g(p, m-w)+g(p, w)
$$


For an arbitrary integer $u$ with $0 \leq u<m$. the relation

$$
g(p, u) \unlhd g(k, u)=f(k, u)
$$

holds. Using the definition of $p$ and (3.4) and (3.8) we have

$$
\begin{aligned}
f(k, m)= & f(p, m)=g(p, m)=g(p, m-w)+ \\
& +g(p, w) \unlhd f(p, m-w)+g(p, w) .
\end{aligned}
$$

Hence by (3.7) and (3.9) and the minimality property of $m$

$$
\begin{aligned}
f(k, m) \unlhd & f(p, m-w)+g(p, w) \unlhd f(k, m-w)+ \\
& +g(k, w)=f(k, m-w)+g\left(k, w-a_{k}\right)+ \\
& +c_{k}=f(k, m-w)+f\left(k, w-a_{k}\right)+c_{k} \unlhd \\
\unlhd & f\left(k, m-a_{k}\right)+c_{k}=g(k, m) \unlhd f(k, m) .
\end{aligned}
$$

Here no equation can hold because of definition $m$, thus we have a contradiction.

The Theorem 3.1 is sharp. Consider the following problem with $n=3$, 4 where $c_{1}=2, c_{2}=2, c_{3}=3, c_{4}=5, a_{1}=1, a_{2}=2, a_{3}=3, a_{4}=6$. If $n=3$ then we have $a_{3}+a_{2}-1=4$ and if $n=4$ then $a_{3}+1=4$ and in both cases $g(3,4)=g(4,4)=$ $=5>f(3,4)=f(4,4)=4$ and this is the only right-hand-side from the interval $\left[a_{n-1}+1, a_{n}+a_{n-1}-1\right]$ where the greedy solution is not optimal.

Theorem 3.2 seems to be stronger since according to it there are only $\left(\begin{array}{l}n \\ 3\end{array}\right)$ candidates for $m$ whose number is independent from the magnitude of the coefficients. But we shall see in the next section that Theorem 3.1 can be efficiently used in proving further theorems.

\section{A Recursive Sufficient Condition of the Pleasantness of the Knapsack Problems}

In this section we always assume that the problem having $n$ variables is pleasant. We add a new variable to the problem and describe the cases when the problem remains pleasant.

The index of the new variable is always supposed to be $n+1$. We claim the regularity conditions for the new problem, too, i.e.:

$$
c_{n+1} \in \mathbb{Z}_{+}, a_{n+1} \in \mathbb{N}, \frac{c_{n}}{a_{n}} \unlhd \frac{c_{n+1}}{a_{n+1}}, a_{n}<a_{n+1} .
$$

Before proving the main theorem of this section, we introduce some notations and prove a theorem which will also be useful in the case of error analysis, too. 
$1: 141,11$ he the eror of the greedy solution in the problem having $n$ varlables and right-hand-side $y, l i c$.

$$
\operatorname{err}(n, y)= \begin{cases}f(n, y)-g(n, y) & \text { in the case of Problem (2.1) } \\ g(n, y)-f(n, y) & \text { in the case of Problem (2.2) }\end{cases}
$$

Theorem 4.1: Assume that (4.1) holds and the problem having $n$ variables is pleasant and consider the problem with $n+1$ variables. Let $i>0$ be an arbitrary integer and $s_{i}$ an integer, such that

$$
i a_{n+1} \leq s_{i} a_{n}<(i+1) a_{n+1} .
$$

Assume that for the integer $y$ the inequality

$$
i a_{n+1} \leq y<(i+1) a_{n+1}
$$

holds and the value of $x_{n+1}^{*}$ is 0 in the $n+1$ variables problem with the right-hand-side $y$. Then

$$
\operatorname{err}\left(n+1, s_{i} a_{n}\right) \geq \operatorname{err}(n+1, y) \text {. }
$$

Proof: First we consider the case

$$
y \geq s_{i} a_{n} .
$$

Let

$$
d=y-s_{i} a_{n}
$$

According to the conditions

$$
f(n+1, y)=f(n, y)=g(n, y)=s_{i} c_{n}+g(n, d) \unlhd f\left(n+1, s_{i} a_{n}\right)+f(n, d) .
$$

Let

$$
m_{i}=s_{i} a_{n}-i a_{n+1} \text {. }
$$

Then

$$
\begin{aligned}
g(n+1, y)= & i c_{n+1}+g\left(n, m_{i}+d\right)=i c_{n+1}+ \\
& +f\left(n, m_{i}+d\right) \unrhd i c_{n+1}+f\left(n, m_{i}\right)+ \\
& +f(n, d)=g\left(n+1, s_{i} a_{n}\right)+f(n, d) .
\end{aligned}
$$

Thus

$$
\begin{aligned}
& g(n+1, y) \unrhd g\left(n+1, s_{i} a_{n}\right)+f(n, d) \\
& f\left(n+1, s_{i} a_{n}\right)+f(n, d) \unrhd f(n+1, y) .
\end{aligned}
$$


Subtracting the two inequalities. we obtain

$$
f\left(n+1, s_{i} a_{n}\right)-g\left(n+1, s_{i} a_{n}\right) \unrhd f(n+1, y)-g(n+1, y\rfloor
$$

This is the desired relation according to the definition of $\unrhd$ and the function err.

Now assume that $y<s_{i} a_{n}$. Let

$$
h=s_{i} a_{n}-y, k=y-i a_{n+1}, d=y-\left(s_{i}-1\right) a_{n}=a_{n}-h .
$$

Consider the number $c_{n}+f(n+1, y)$. From the conditions of the theorem we obtain

$$
\begin{aligned}
c_{n}+f(n+1, y) & =c_{n}+f(n, y)=c_{n}+g(n, y)= \\
& =c_{n} s_{i}+f(n, d) \unlhd f\left(n, s_{i} a_{n}\right)+f(n, d) .
\end{aligned}
$$

Similarly

$$
\begin{aligned}
c_{n}+g(n+1, y)= & c_{n}+i c_{n+1}+f(n, k)=i c_{n+1}+ \\
& +f\left(n, a_{n}+k\right)=i c_{n+1}+f(n, h+k+d) \unrhd \\
\unrhd & i c_{n+1}+f(n, h+k)+f(n, d)= \\
= & g\left(n+1, s_{i} a_{n}\right)+f(n, d) .
\end{aligned}
$$

Subtracting again the two inequalities, we obtain the desired relation.

Theorem 4.2: Assume that the regularity conditions with (4.1) are satisfied and the problem having $n$ variables is pleasant. Let $s$ and $t$ be the integers

$$
s=\left\lceil\frac{a_{n+1}}{a_{n}}\right\rceil, \quad t=s a_{n}-a_{n+1} .
$$

Then the following two statements are equivalent:

(i) the problem having $n+1$ variables is pleasant,

(ii) $c_{n+1}+g(n, t) \unrhd s c_{n}$.

Proof: According to Theorem 3.1, the problem having $n+1$ variables is pleasant if and only if for any integer satisfying the inequality

$$
a_{n}+1 \leq y \leq a_{n+1}+a_{n}-1
$$

the equation

$$
f(n+1, y)=g(n+1, y)
$$

holds. If $y<a_{n+1}$ then

$$
f(n+1, y)=f(n, y)=g(n, y)=g(n+1, y) .
$$

It follows from the regularity conditions and (4.1) that

$$
f\left(n+1, a_{n+1}\right)=c_{n+1}=g\left(n+1, a_{n+1}\right) .
$$


Inumune

$$
a_{n}+a_{n-1}-1 \geq y>a_{n}
$$

we have two cases according to the two possible optimal values of $x_{n+1}$. If $x_{n+1}=1$ in at least one optimal solution then

$$
f(n+1, y)=c_{n+1}+f\left(n, y-a_{n+1}\right)=c_{n+1}+g\left(n, y-a_{n+1}\right)=g(n+1, y) .
$$

If $x_{n+1}=0$ in every optimal solution then according to the previous theorem

$$
\operatorname{err}(n+1, y) \leq \operatorname{err}\left(n+1, s a_{n}\right) \text {. }
$$

Thus the problem is pleasant if and only if

$$
\operatorname{err}\left(n+1, s a_{n}\right)=0 .
$$

The best objective function value, which can be achieved if $x_{n+1}=1$, with the right-hand-side $s a_{n}$ is

$$
c_{n+1}+f(n, t)=c_{n+1}+g(n, t)^{\prime}=g\left(n+1, s a_{n}\right) .
$$

Similarly it follows from the regularity conditions that

$$
S c_{n}
$$

is the best objective function value which can be achieved if $x_{n+1}=0$. Thus (4.3) holds if and only if

$$
c_{n+1}+g(n, t) \unrhd s c_{n} .
$$

Condition (ii) can be checked very easily. It is always necessary for the pleasantness but is sufficient only in the case where the functions $f(n, \cdot)$ and $g(n, \cdot)$ are identical. This is illustrated by the following example: Consider the Problem (2.2) with $n=3$ and $c_{1}=c_{2}=c_{3}=c_{4}=1, a_{1}=1, a_{2}=3, a_{3}=4, a_{4}=7$. Here $s=2$ and $8=7+1=2 \cdot 4$, thus the value of both sides of (ii) is 2 . But

$$
g(4,6)=g(3,6)=3>f(4,6)=2 .
$$

\section{Error Analysis}

In the previous section we have described the cases where after adding a new variable to the problem, it remains pleasant. Now we determine its maximal error if it fails to be pleasant. 


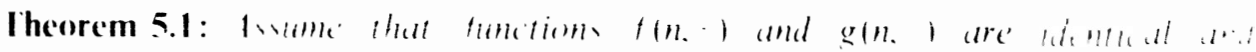
consider the problem having $n+1$ variables. For every right-hand-side $y$ we have a right-hand-side $z \in \mathbb{Z}_{+}$, such that

$$
z \leq y, \operatorname{err}(n+1, z)=\operatorname{err}(n+1, y)
$$

and the value of the variable $x_{n+1}$ is 0 in at least one optimal solution belonging to $z$.

Proof: Let $x^{*}$ be an optimal solution belonging to $y$ and having a maximal value in the $n+1$-th component. Let

$$
z=y-a_{n+1} x_{n+1}^{*} \text {. }
$$

Then clearly $z \geq 0$ and

and

$$
g(n+1, y)=c_{n+1} x_{n+1}^{*}+g(n+1, z)
$$

$$
f(n+1, y)=c_{n+1} x_{n+1}^{*}+f(n+1, z) .
$$

Subtracting the two equations we obtain the desired relation.

Theorem 5.2: Assume that (4.1) is fulfilled. Let $0<i<j$ be two arbitrary integers. Assume that $s_{i}$ and $s_{j}$ are integers satisfying the inequalities

$$
i a_{n+1} \leq s_{i} a_{n}<(i+1) a_{n+1}, j a_{n+1} \leq s_{j} a_{n}<(j+1) a_{n+1} .
$$

Suppose that

Then

$$
s_{i} a_{n}-i a_{n+1}=s_{j} a_{n}-j a_{n+1} .
$$

$$
i c_{n+1}-s_{i} a_{n} \unlhd j c_{n+1}-s_{j} c_{n} \text {. }
$$

Proof: We obtain from (5.1) and (4.1) that

$$
\frac{s_{j}-s_{i}}{j-i}=\frac{a_{n+1}}{a_{n}} \unlhd \frac{c_{n+1}}{c_{n}} .
$$

Hence we get the desired relation by multiplying the sides with the positive number $(j-i) c_{n}$.

Theorem 5.3: Assume that the regularity conditions including (4.1) are satisfied and the functions $f(n, \cdot)$ and $g(n, \cdot)$ are identical. Let

and

$$
d=\text { g.c.d. }\left(a_{n}, a_{n+1}\right)
$$

and

$$
s_{i}=\left[\begin{array}{c}
i a_{n+1} \\
a_{n}
\end{array}\right], t_{i}=s_{i} a_{n}-i a_{n+1}, i \in \mathbb{N},
$$

$$
\delta=\left\{\begin{aligned}
1 & \text { in the case of Problem (2.1) } \\
-1 & \text { in the case of Problem (2.2) }
\end{aligned}\right.
$$




$$
\begin{aligned}
& \max \left\{\operatorname{err}(n+1, y): y \in \mathbb{Z}_{+}\right\}= \\
= & \max \left\{\max \left\{0, \delta\left(s_{i} c_{n}-i c_{n+1}-g\left(n, t_{i}\right)\right)\right\}: i=1, \ldots,\left(a_{n} / d\right)-1\right\} .
\end{aligned}
$$

Proof: It follows from Theorem 5.1 and 4.1 that

$$
\max \left\{\operatorname{err}(n+1, y): y \in \mathbb{Z}_{+}\right\}=\max \left\{\operatorname{err}\left(n+1, s_{i} a_{n}\right): i \in \mathbb{N}\right\} .
$$

We obtain from the definition of the numbers $d$ an $t_{i}$ that

$$
\left\{t_{i}: i=1, \ldots,\left(a_{n} / d\right)\right\}=\left\{0, d, 2 d, \ldots, a_{n}-d\right\} .
$$

It follows from the regularity conditions that if $i a_{n+1}=s_{i} a_{n}$, i.e., $t_{i}=0$, then $\operatorname{err}\left(n+1, s_{i} a_{n}\right)=0$. This situation occurs first at $i=a_{n} / d$. Thus we obtain from Eq. (5.3) by Theorem 5.2 that

$$
\max \left\{\operatorname{err}(n+1, y): y \in \mathbb{Z}_{+}\right\}+=\max \left\{\operatorname{err}\left(n+1, s_{i} a_{n}\right): i=1, \ldots,\left(a_{n} / d\right)-1\right\} .
$$

Now we consider the solutions determining the value $f\left(n+1, s_{i} a_{n}\right)$. If $x_{n+1}=0$ at least in one such solution then $x_{1}=\ldots=x_{n-1}=x_{n+1}=0, x_{n}=s_{i}$ is an optimal solution and hence

$$
\operatorname{err}\left(n+1, s_{i} a_{n}\right)=\delta\left(s_{i} c_{n}-i c_{n+1}-m\left(n, t_{i}\right)\right)
$$

If $x_{n+1}>0$ in every optimal solution determining $f\left(n+1, s_{i} a_{n}\right)$ then we have an integer $k$ with $1 \leq k<i$ and a right-hand-side $y$ with

$$
y=s_{i} a_{n}-k a_{n+1}
$$

such that

$$
\operatorname{err}\left(n+1, s_{i} a_{n}\right)=\operatorname{err}(n+1, y) \leq \operatorname{err}\left(n+1, s_{i-k} a_{n}\right)
$$

If $f\left(n+1, s_{i-k} a_{n}\right)$ has no optimal solution with $x_{n+1}=0$ then we can repeat this procedure. Finally we obtain that the maximum in

$$
\max \left\{\operatorname{err}\left(n+1, s_{i} a_{n}\right): i=1, \ldots,\left(a_{n} / d\right)-1\right\}
$$

is achieved at a value $j$, such that we have at least one optimal solution determining $f\left(n+1, j a_{n}\right)$ with $x_{n+1}=0$. Hence we obtain the statement from (5.3). 


\section{Conclusions}

In this paper a unified discussion has been expounded to some closely related results which are independently achieved in number theory and integer programming and have been partly generalized. The Theorems 3.1 and 3.2 have been proved in [7] and [3] respectively, for Problem (2.2) with $c_{1}=\ldots=c_{n}=1$. The first result of the type of Theorem 4.2 has been obtained in [1] for the same problem.

The appropriate theorem for Problem (2.2) with an arbitrary objective function has been found independently in [4]. MARSTANDER has obtained the same result for Problem (2.1). Both of the proofs in [4] and [5] are appropriate for a unified discussion with the notations $\unrhd$ and $\unlhd$. Here a shorter proof is given using Theorem 3.1. Error analysis has been found only for Problem (2.2) in $[4]$.

\section{References}

[1] Djawad, M.: Kennzeichnung von Mengen mit einer additiven Minimalcigenschaft. J. reino angew. Math., 311312 (1979), also in der Dissertation, Math. Inst. Joh. Gutenberg-Univ., Mainz 1974.

[2] Girlich, E.; M. M. Kowaljow: Nichtlineare diskrete Optimierung. Mathematical Research/ Mathematische Forschung, Vol. 6., Akademie-Verlag, Berlin, 1981.

[3] Johnson, S. C.; B. W. Kerningham: Making change with a minimum number of coins manuscript. (undated), Bell Telephone Laboratories. Murray Hill, New Jersey.

[4] Magazine, M. J.; G. L. Nemhauser; L. E. Trotter JR.: “Then the Greedy Solution Solves a Class of Knapsack Problems”, Operations Research, 24 (1975) 207-217.

[5] Marstander, O.: On a Problem of Frobenius. Math. Scand., 58 (1986) 161 - 175.

[6] Selmer, E. S.: The Local Postage Stamp Problem. University of Bergen, Norway, Dept. of Pure Math., No. 42-04-15-86.

[7] ZöLlNer, J.: Über angenehme Mengen. Mainzer Seminarberichte in additiver Zahlentheorie, 1 (1983) $53-71$.

Received October 1990, revised April 1991

B. VIZVÁRI

Bilkent University

Dept. of Industrial Engineering

06533 Bilkent, Ankara

Turkey 